\title{
Avaliação das oportunidades de desenvolvimento motor na habitação familiar de crianças entre 18 e 42 meses
}

\author{
Assessment of opportunities for motor development in the family home \\ of children between 18 and 42 months of age
}

\author{
L.G. Giordani, C.S. Almeida, A.M. Pacheco
}

ARTIGO ORIGINAL | ORIGINAL ARTICLE

\begin{abstract}
RESUMO
O trabalho teve o objetivo de avaliar as oportunidades para o desenvolvimento motor na habitação familiar de crianças entre 18 e 42 meses, comparando as faixas etárias (18 a 30 meses e 31 a 42 meses), os gêneros, o convívio com outras crianças e as condições socioeconômicas. Caracterizou-se como um estudo observacional, descritivo, do tipo transversal. Participaram do estudo 88 crianças da cidade de Sapucaia do Sul/RS. Foi utilizado o questionário Affordances in the Home Environment Motor Development em crianças de 18 - 42 meses, durante visita domiciliar. Não houve diferença entre o gênero e as faixas etárias. Verificou-se que o número de adultos estavam associados com melhor escore de espaço interior. A escolaridade dos pais associou-se com maior oferta de brinquedos e materiais de motricidade fina $(p$ $<$.005). O número de crianças no domicílio estava associado com uma melhor variedade de estimulação $(p<.001)$. A renda familiar estabeleceu relação significativa com o espaço interior $(p<.005)$, espaço exterior $(p<.001)$, materiais de motricidade fina $(p<.005)$ e de motricidade ampla $(p<.001)$. A criança que apresentou convívio social diário teve melhor variedade de estímulo $(p<.001)$. Contudo, é importante incrementar a atenção à criança e orientar seus responsáveis acerca do desenvolvimento. Palavras-chave: desenvolvimento infantil, ambiente, jogos e brinquedos
\end{abstract}

ABSTRACT

This study aimed to assess the opportunities for motor development in the family home of children that are between 18 and 42 months old, comparing the age groups (18 - 30 months and 31 - 42 months), gender, living with children and other conditions socioeconomic Characterized as an observational, descriptive and transversal study. The study included 88 children from the city of Sapucaia do Sul/ RS. Was used a structured and validated previously questionnaire called Affordances in the Home Environment Motor Development of children that are between 18 - 42 months, during home visits. There was no difference between gender and age groups. It was found that the number of adults are associated with better scores of interior space. The parents' education is associated with greater supply of toys and materials of skills fine motor $(p<.005)$. The number of children at home is associated with a better variety of stimulation $(p<.001)$. Family income has a significant relationship with the interior space $(p<.005)$, exterior space $(p<.001)$, materials of skills fine motor $(p<.005)$ and gross motor $(p<.001)$. The child who had more daily social interaction has more variety of stimulation $(p$ $<.001)$. However, it is important to increase attention to children and advising their parents about the development.

Keywords: child development, environment, toys

Submetido: 08.08.2012 | Aceite: 15.05.2013

Letícia Gue Giordani. Unisinos, Brasil.

Carla Skilhan Almeida, Adriana Moré Pacheco. Universidade Federal do Rio Grande do Sul, Brasil.

Endereço para correspondência: Adriana Moré Pacheco, Escola de Educação Física - ESEF/UFRGS, Rua Felizardo,

750, Jardim Botânico, Porto Alegre, RS, CEP: 90690-200, Brasil.

E-mail: adrimpacheco@ufrgs.br 
Desenvolvimento motor é definido como um processo permanente de alteração no nível de funcionamento de um indivíduo. Isso significa que, no decorrer da vida, são necessários ajustes, compensações ou mudanças a fim de obter ou manter alguma habilidade. Ele abrange as necessidades biológicas, ambientais e ocupacionais, além das habilidades motoras. A aquisição de alguma habilidade irá depender da exigência da tarefa, resultando da interação entre o indivíduo (fatores biológicos, hereditariedade) e o ambiente (fatores de experiência e aprendizagem). Esses fatores podem ser transformados, um pelo outro (Gallahue \& Ozmun, 2005).

Nicoletti e Manoel (2007) acrescentam que o desenvolvimento motor constitui um conjunto de mudanças que capacita o indivíduo a interagir com os meios físico e social, não envolvendo apenas mudanças nos movimentos, mas também nas relações e objetivos relacionados com o ambiente. Tipicamente, existe uma sequência na aquisição das habilidades. Um bebê normal, por exemplo, consegue engatinhar, caminhar e depois correr em certa etapa do desenvolvimento. A hereditariedade estabelece as bases para o desenvolvimento, porém, o ambiente/experiência é quem afeta o ritmo e a aquisição das habilidades motoras. Além disso, o ambiente desempenha importante papel no desenvolvimento da linguagem enquanto a cognição e a personalidade são mais influenciadas por variações de experiência (Gabbard, Caçola, \& Rodrigues, 2008).

Ao contrário do que muitos acreditam, a maioria das crianças não aprende os movimentos fundamentais "automaticamente". O aprendizado envolve uma série de fatores, tais como, oportunidades práticas, encorajamento, instruções e condições ambientais para realizar a tarefa (Gallahue \& Ozmun, 2005). A investigação do desenvolvimento infantil sugere que um ótimo nível de desenvolvimento acontece em ambientes favoráveis. Nesse sentido, a habitação familiar constitui um agente primordial para a aprendizagem. As oportunidades ofere- cidas à criança é que potencializam o desenvolvimento das habilidades (Gabbard et al., 2008; Rodrigues, Saraiva, \& Gabbard, 2005). $\mathrm{Na}$ primeira infância, os principais vínculos, bem como os cuidados e estímulos necessários ao crescimento e desenvolvimento, são fornecidos pela família. A qualidade do cuidado, nos aspectos físico e afetivo-social, decorre de condições estáveis de vida, tanto socioeconômicas quanto psicossociais (Andrade et al., 2005). O ambiente familiar é um dos agentes primordiais para o desenvolvimento infantil. Apesar disso, existe pouca investigação acerca dessa situação (Rodrigues et al., 2005).

O objetivo do presente estudo foi avaliar as oportunidades para o desenvolvimento motor na habitação familiar de crianças entre 18 e 42 meses, comparando as faixas etárias (18 a 30 meses e 31 a 42 meses), os gêneros, o convívio com outras crianças e as condições socioeconômicas.

\section{MÉTODO}

Estudo observacional, descritivo, do tipo transversal realizado com crianças em idades entre 18 e 42 meses, independente da raça, de ambos gêneros, residentes nas Vilas Colonial e Fortuna que são atendidas pelo programa de Fisioterapia Comunitária da Universidade do Vale do Rio dos Sinos - Unisinos, localizadas na cidade de Sapucaia do Sul/RS.

\section{Amostra}

A população referência do estudo foi de 139 indivíduos [levantamento realizado em conjunto com os Agentes Comunitários de Saúde (ACS)]. Cada um dos postos atendia a seis micro-áreas. Das 139 crianças mapeadas para a avaliação do presente estudo, apenas 88 finalizaram o processo. Isso aconteceu devido a alguns fatores como: falta de identificação das moradias, crianças que alternavam as residências (casa dos pais, avós), mudança de endereço e recusa por parte do responsável.

O presente estudo passou pela aprovação 
do Comitê de Ética da Universidade que deu origem ao estudo, seguindo os parâmetros éticos da resolução 196/96, do Conselho Nacional da Saúde sob o protocolo de $n^{\circ}$ 08/061. Os participantes assinaram o Termo de Consentimento Livre e Esclarecido (TCLE) em duas vias e foram submetidos a um questionário estruturado e previamente validado com a finalidade de obter informações inerentes a pesquisa.

\section{Instrumentos}

$\mathrm{O}$ instrumento utilizado foi o Affordances in the Home Environment Motor Development (AHEMD) traduzido para o português como Oportunidades de Estimulação Motora na Casa Familiar. É considerado um instrumento de avaliação de fácil aplicação e definido para cada faixa etária. A versão utilizada foi a 18 - 42 meses (Rodrigues et al., 2005). O questionário é composto por 67 itens que contempla a caracterização da criança e da família, espaço físico da residência (interior e exterior), atividades diárias e brinquedos e materiais existentes na habitação. Inclui perguntas com respostas dicotômicas, escala Likert e descritivas, conforme exemplo (Figura 1).

A organização do questionário é baseada em cinco subescalas: espaço exterior, espaço interior, variedade de estimulação, materiais de motricidade fina e materiais de motricidade ampla. Além das pontuações de cada subescala foi construído um indicador global denominado AHEMD total, constituído pela soma dos anteriores. Quanto maior a pontuação, maiores eram as oportunidades de desenvolvimento motor.

\section{Procedimentos}

O instrumento foi aplicado sempre pelo mesmo pesquisador durante visita ao domicílio das famílias, de forma oral, com o objetivo de manter um padrão de resposta e oferecer alternativas para o levantamento do número de materiais.

Exemplo de questão dicotômica

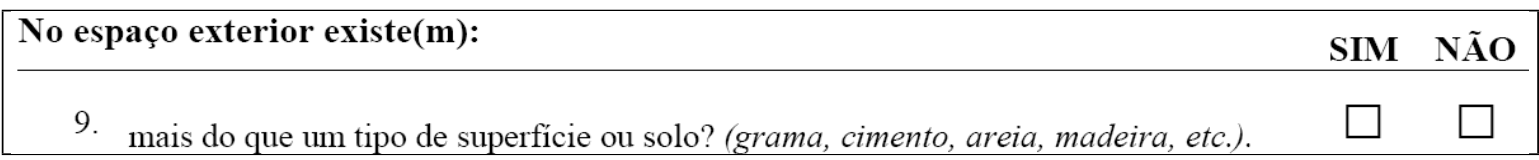

Exemplo de questão em escala Likert

Num dia típico, como descreveria a quantidade de tempo que o seu filho (a) passa acordado em cada uma das situações abaixo descritas? (Leia cada questão cuidadosamente e marque a opção que melhor descreve a sua resposta)

33. Carregado por adultos no colo, ou em algum dispositivo de transporte (mochila porta-bebê/ bebê bag etc).

$$
\text { Quase Nunca } \square \quad \text { Pouco Tempo } \square \quad \text { Muito Tempo } \square \quad \text { Quase Sempre } \square
$$

Exemplo de questão descritiva

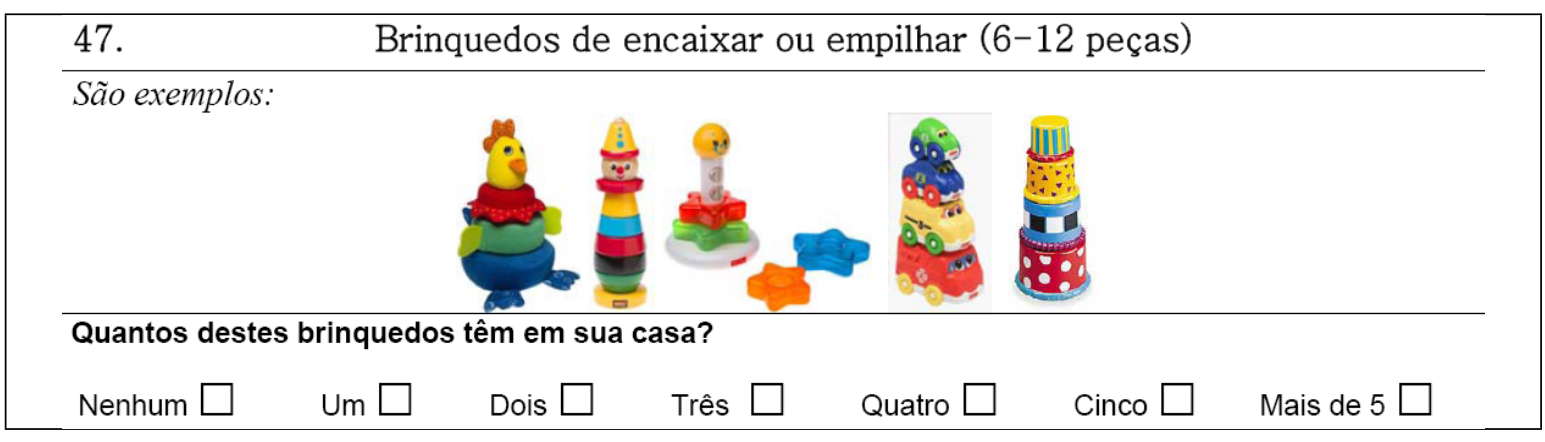

Figura 1. Categorias de questões encontradas no AHEMD. 


\section{Análise Estatística}

Os dados categóricos foram descritos através de frequências e percentuais. As variáveis quantitativas foram descritas pela média \pm desvio padrão da média. A comparação entre os achados conforme gênero e faixa etária foi realizada através do teste $t$ de student para amostras independentes. A associação entre o convívio com outras crianças e nível socioeconômico com os escores das subescalas do AHEMD foram avaliados através do coeficiente de correlação de Spearman. A associação entre as variáveis categóricas foi avaliada através do teste qui-quadrado de Pearson. O nível de significância adotado no estudo foi de $p \leq .05$. Os dados foram processados e analisados com o auxílio dos programas Microsoft Office Excel 2007 e Statistical Package for Social Science (SPSS) para Windows, versão 18.0.

\section{RESULTADOS}

Das 88 crianças estudadas, $50 \%$ eram meninos $(n=44)$, tinham idade entre 18 e 42 meses, sendo que a média de idade foi de 30.40 meses. Não houve diferença estatisticamente significativa $(p=.182)$ entre os gêneros e entre as faixas etárias observadas (18 a 30 meses e 31 a 42 meses). Os pais das crianças, em sua maioria, estudaram até o Ensino Fundamental ( $5^{\mathrm{a}}$ a $8^{\mathrm{a}}$ série) e a renda familiar mensal para $60.2 \%$ da amostra era inferior a $\mathrm{R} \$ 1.000 .00$. O escore médio da amostra para as oportunidades de desenvolvimento na habitação familiar foi de $10.30 \pm 1.90$, próximo do limite inferior para a classificação adequada (para o AHEMD total, escore maior que 10). Na Tabela 1 podem-se observar as frequências, médias e desvios-padrão que qualificam a amostra do estudo.

Na Tabela 2 observa-se a distribuição da classificação de acordo com as subescalas do questionário AHEMD: espaço interior, espaço exterior e variedade de estimulação. A classificação mais freqüente para interior foi muito boa $(68.2 \%)$, para exterior boa $(55.7 \%)$ e para variedade de estimulação muito boa (51.1\%).
$\mathrm{Na}$ Tabela 3 estão descritas as associações entre as variáveis. Pode-se observar que o número de adultos e de quartos está associado com melhor qualidade do espaço interior. A renda apresenta-se relacionada tanto com a qualidade do ambiente interior e exterior como com a oferta de materiais e brinquedos de motricidade fina e ampla. Quanto

Tabela 1

Caracterização da amostra

\begin{tabular}{|c|c|}
\hline Variáveis & $n=88$ \\
\hline \multicolumn{2}{|l|}{ Gênero - $n(\%)$} \\
\hline Masculino & $44(50.0)$ \\
\hline Feminino & $44(50.0)$ \\
\hline Idade- Média \pm DP & $30.4 \pm 7.2$ \\
\hline Número de adultos - Média \pm DP & $2.6 \pm 0.9$ \\
\hline Número de crianças - Média \pm DP & $1.9 \pm 1.0$ \\
\hline Número de quartos - Média \pm DP & $2.3 \pm 0.9$ \\
\hline \multicolumn{2}{|l|}{ Escolaridade do pai - $n(\%)$} \\
\hline Educação infantil & $12(13.6)$ \\
\hline Ensino fundamental & $42(47.7)$ \\
\hline Ensino médio & $31(35.2)$ \\
\hline Ensino técnico/superior & $3(3.4)$ \\
\hline \multicolumn{2}{|l|}{ Escolaridade da mãe - $n(\%)$} \\
\hline Educação infantil & $9(10.2)$ \\
\hline Ensino fundamental & $51(58.0)$ \\
\hline Ensino médio & $24(27.3)$ \\
\hline Ensino técnico/superior & $4(4.5)$ \\
\hline \multicolumn{2}{|l|}{ Renda familiar - $n(\%)$} \\
\hline Menos de $\mathrm{R} \$ 1.000 .00$ & $53(60.2)$ \\
\hline $\mathrm{R} \$ 1.000,00$ a $\mathrm{R} \$ 1.500 .00$ & $19(21.6)$ \\
\hline $\mathrm{R} \$ 1.500,00$ a $\mathrm{R} \$ 2.500 .00$ & $11(12.5)$ \\
\hline $\mathrm{R} \$ 2.500,00$ a $\mathrm{R} \$ 3.500 .00$ & $5(5.7)$ \\
\hline AHEMD total- Média $\pm \mathrm{DP}$ & $10.3 \pm 1.9$ \\
\hline
\end{tabular}

Nota: DP $=$ desvio padrão

Tabela 2

Questionário AHEMD

\begin{tabular}{cc}
\hline Subescala (escore AHEMD) & $n(\%)$ \\
\hline Espaço Interior & $7(8.0)$ \\
Muito fraco (1) & $8(9.1)$ \\
Fraco (2) & $1(14.8)$ \\
Bom (3) & $60(68.2)$ \\
Muito Bom (4) & \\
Espaço Exterior & $15(17.0)$ \\
Muito fraco (1) & $10(11.4)$ \\
Fraco (2) & $49(55.7)$ \\
Bom (3) & $14(15.9)$ \\
Muito Bom (4) & \\
Variedade de Estimulação & $2(2.3)$ \\
Muito fraca (1) & $18(20.5)$ \\
Fraca (2) & $23(26.1)$ \\
Boa (3) & $45(51.1)$ \\
Muito Boa (4)
\end{tabular}


maior o número de adulto no domicílio, mais prejudicado era a variedade de estimulação das crianças. A presença de outras crianças melhora a variedade de estimulação. A oferta de materiais de motricidade fina está associada com a escolaridade dos pais.

Dentre os materiais ofertados as crianças a maioria consiste em brinquedos de faz de conta (42.0\%) e materiais educacionais (22.0\%). Se observarmos apenas o gênero feminino, o primeiro sobe para $44.0 \%$. Realmente este número é ainda maior, visto que o questionário classifica até cinco itens em cada tipo de brinquedo (seis questões referentes a brinquedos de faz de conta). Existe uma grande carência de materiais de montar/encaixar/empilhar (classificados como puzzles e materiais de construção) visto que apenas 22 meninos e 20 meninas do total de 88 crianças possuem esse tipo de brinquedo em suas residências totalizando $2.0 \%$ dos materiais encontrados. Isso talvez aconteça devido ao menor apelo comercial para esse tipo de material. Foram identificadas poucas ocorrências de materiais violentos, (arma de fogo, espada) e os mesmos não foram classificados porque não contemplaram a descrição do questionário utilizado. Materiais musicais apresentaram uma freqüência de $15.0 \%$, materiais locomotores $7.0 \%$, materiais manipulativos $6.0 \%$ e materiais de exploração global 4.0\%.

$\mathrm{Na}$ Tabela 4, apresenta-se a associação do escore de AHEMD com convívio social. Observa-se que a criança que apresentou convívio social diário teve melhor variedade de estímulo $(p=.001)$.

\section{DISCUSSÃO}

Na primeira infância, a qualidade da relação com outras pessoas, além da manutenção dos processos orgânicos, favorece a aquisição de habilidades. O desenvolvimento infantil é favorecido pela genética, mas as potencialidades só se desenvolvem na medida em que existe um meio favorável físico e emocionalmente. É através das relações que a criança se descobre, constrói a sua personalidade (Andrade et al., 2005).

Um dos principais elementos para uma adequada estimulação no ambiente familiar é o convívio da criança com adultos ou até mesmo outras crianças. O convívio familiar possibilita que a criança desenvolva sua percepção e comportamento, adquira conhecimentos e habilidades e estabeleça relações no seu microssistema, reconhecendo-se como parte integrante dele (Andrade et al., 2005; Bronfenbrenner, 2002).

A família, além de ser responsável pelos cuidados, deve proporcionar os estímulos necessários para o crescimento e desenvolvimento infantil. Além disso, deve garantir à criança as necessidades fisiológicas, afetivas, cognitivas, além de segurança e estabilidade (Andrade et al., 2005).

Atualmente existem novos arranjos de família diferentes da nuclear, constituída por casal e filhos, dominante tempos atrás. A

Tabela 3

Associação entre as variáveis

\begin{tabular}{cccccc}
\hline & Interior* & Exterior* & $\begin{array}{c}\text { Variedade de } \\
\text { Estimulação* }\end{array}$ & MF & MG \\
& $R s(p)$ & $R s(p)$ & $R s(p)$ & $R s(p)$ & $R s(p)$ \\
\hline $\mathrm{N}^{\circ}$ adultos & $0.421(<.001)$ & $0.126(.242)$ & $-0.281(<.001)$ & $-0.052(.634)$ & $-0.069(.524)$ \\
$\mathrm{N}^{\circ}$ crianças & $0.070(.514)$ & $0.074(.491)$ & $0.474(<.001)$ & $-0.306(<.005)$ & $-0.151(.161)$ \\
$\mathrm{N}^{\circ}$ quartos & $0.675(<.001)$ & $0.212(<.005)$ & $-0.005(.967)$ & $-0.049(.651)$ & $-0.056(.603)$ \\
Escolaridade & & & & & \\
Pai & $0.072(.508)$ & $-0.089(.412)$ & $0.030(.780)$ & $0.227(<.005)$ & $0.078(.468)$ \\
Mãe & $0.054(.616)$ & $0.174(0,104)$ & $-0.123(.254)$ & $0.244(<.005)$ & $0.193(.072)$ \\
Renda & $0.220(<.005)$ & $0.280(<.001)$ & $-0.105(.330)$ & $0.258(<.005)$ & $0.386(<.001)$ \\
\hline
\end{tabular}

Nota: $\mathrm{MF}=$ Motricidade Fina; $\mathrm{MG}=$ Motricidade Ampla; Rs = Coeficiente de Correlação de Spearman

* Classificado como valor estandardizado

** Número de materiais 
Tabela 4

Associação do escore de AHEMD com convívio social

\begin{tabular}{|c|c|c|c|}
\hline \multirow[b]{2}{*}{ AHEMD } & \multicolumn{2}{|c|}{ Convívio Social } & \multirow[b]{2}{*}{$p$ valor } \\
\hline & $\operatorname{Sim}(n=48)$ & Não $(n=40)$ & \\
\hline \multicolumn{4}{|l|}{ Interior $-n(\%)$} \\
\hline Muito fraco & $4(8.3)$ & $3(7.5)$ & \multirow{4}{*}{$.603^{*}$} \\
\hline Fraco & $5(10.4)$ & $3(7.5)$ & \\
\hline Bom & $9(18.8)$ & $4(10.0)$ & \\
\hline Muito Bom & $30(62.5)$ & $30(75.0)$ & \\
\hline \multicolumn{4}{|l|}{ Exterior $-n(\%)$} \\
\hline Muito fraco & $8(16.7)$ & $7(17.5)$ & \multirow{4}{*}{$.059 *$} \\
\hline Fraco & $6(12.5)$ & $4(10.0)$ & \\
\hline Bom & $22(45.8)$ & $27(67.5)$ & \\
\hline Muito Bom & $12(25.0)$ & $2(5.0)$ & \\
\hline \multicolumn{4}{|c|}{ Variedade de Estimulação - $n(\%)$} \\
\hline Muito fraca & $0(0.0)$ & $2(5.0)$ & \multirow{4}{*}{$<.001^{*}$} \\
\hline Fraca & $2(4.2)$ & $16(40.0)$ & \\
\hline Boa & $9(18.8)$ & $14(35.0)$ & \\
\hline Muito Boa & $37(77.1)$ & $8(20.0)$ & \\
\hline MF- Média \pm DP & $18.06 \pm 7.4$ & $18.08 \pm 8.5$ & $.994^{* *}$ \\
\hline MG- Média \pm DP & $8.81 \pm 4.6$ & $8.40 \pm 5.1$ & $.691^{* *}$ \\
\hline \multicolumn{4}{|l|}{ AHEMD total $-n(\%)$} \\
\hline Baixa & $10(20.8)$ & $19(47.5)$ & \multirow[t]{2}{*}{$.015^{*}$} \\
\hline Média & $38(79.2)$ & $21(52.5)$ & \\
\hline
\end{tabular}

Nota: $D P=$ desvio padrão; $M F=$ Motricidade Fina; $M G=$ Motricidade Ampla

* Teste Qui-Quadrado de Pearson

** Teste $t$

família, independentemente de como se estrutura, desempenha o papel de mediadora entre a criança e a sociedade, constituindo um meio relacional (Bronfenbrenner, 2002). O fato observado nos resultados de quanto maior o número de adultos na residência, menor a variedade de estimulação, provavelmente, é devido à aglutinação das formações familiares. Por exemplo: uma família constituída pelo pai, mãe e dois filhos adolescentes. Um dos filhos engravida a namorada e a mesma vem com o seu filho para morar na casa dos avós. A casa passa a ter cinco adultos e uma criança, constituindo dessa maneira, possivelmente, um ambiente com menor variedade de estimulação.

Outra informação importante é de que a presença de companheiro no ambiente familiar interfere positivamente na variedade de estimulação. Isso pode estar relacionado também ao desempenho da função materna. As condições de saúde na infância são influenciadas pela progressão da renda familiar e crescente escolaridade de seus membros. Quanto maior o nível de escolaridade, melhores poderão ser as oportunidades de emprego, o que resultará em melhor renda e oportunidade de oferecer estímulos adequados para favorecer o desenvolvimento da criança. A escolaridade, por sua vez, exerce influência sobre as oportunidades de emprego, dessa forma, condicionando o poder aquisitivo das famílias (Maria-Mengel \& Linhares, 2007; Monteiro \& Freitas, 2000).

Existe uma constante associação entre a qualidade do ambiente e o desenvolvimento das crianças. Alguns fatores de risco, tais como, baixo peso ao nascer, desnutrição, baixa renda familiar, baixa escolaridade dos pais e famílias muito numerosas exercem influência na qualidade do ambiente e desenvolvimento infantil (Müller, 2008; Schobert, 2008).

O ambiente exerce um papel fundamental no desenvolvimento das crianças. Um nível ótimo de desenvolvimento é identificado, principalmente, em contextos com suporte e estimulação adequada (Rodrigues et al., 2005).

Vários estudos apontam que a escolaridade materna funciona como um fator de proteção para o desenvolvimento das crianças (Andrade 
et al., 2005; Martins, Costa, Saforcada, \& Cunha, 2004). No presente estudo não houve associação significativa entre a escolaridade materna em quatro estratos e o escore do questionário. Talvez isso seja devido à característica do local da amostra. A presente pesquisa foi realizada em comunidades carentes, na periferia da cidade. Outros estudos analisaram em áreas centrais e periféricas, talvez por este motivo tenham encontrado associação (Andrade et al., 2005; Martins et al., 2004).

Estudo longitudinal realizado por Martins, Costa, Saforcada e Cunha (2004), na cidade de Pelotas/RS, utilizou o inventário Home Observation for the Measurement of the Environment (HOME) e encontrou associação entre a menor escolaridade dos pais e diminuição da qualidade do ambiente. Os ambientes negativos também estavam associados ao número elevado de irmãos (quatro ou mais) e à prática de dormir com os pais. Apesar disso, mais do que o grau de instrução, o comportamento afetivo, a estabilidade do relacionamento e os cuidados dispensados à criança são fatores de proteção para o desenvolvimento infantil (Martins et al., 2004).

Estudo realizado por Andrade et al. (2005) em 350 crianças com idade entre 17 e 42 meses utilizando o inventário HOME verificou que o nível de escolaridade materna estava associado positivamente com a qualidade de estimulação ambiental. Além disso, quando superior a cinco anos de estudo estava associada também à melhor organização do ambiente físico e temporal, a maior variação na estimulação diária, disponibilidade de materiais e jogos apropriados e maior envolvimento emocional e verbal da mãe com a criança (Andrade et al., 2005).

Conforme a teoria ecológica, a escolaridade e ocupação dos pais interferem diretamente nas relações sociais proximais que ocorrem no microssistema familiar, influenciando, dessa maneira, o desenvolvimento das crianças (Bronfenbrenner, 2002; Maria-Mengel \& Linhares, 2007). No contexto da pesquisa realizada, é pertinente afirmar que muitas crianças da comunidade convivem com situações de riscos psicossociais constantemente. Isso abrange tanto a baixa escolarização quanto a renda familiar insuficiente para prover recursos e materiais básicos para o sustento da família, afetando, dessa forma, o desenvolvimento dos sujeitos.

O brincar constitui uma forma complexa e indispensável ao desenvolvimento infantil (Aranega Nassim, \& Chiappetta, 2006). O brinquedo estimula a criança e a convida para determinada atividade. É a partir da brincadeira que a criança consegue se comparar com os outros (Cordazzo \& Vieira, 2007).

A brincadeira constitui uma atividade espontânea que permite condições apropriadas para o desenvolvimento biopsicossocial da criança. O brinquedo favorece o desenvolvimento infantil em todas as dimensões, incluindo aspectos físicos e sensoriais (percepção, habilidade motora, força, resistência, termorregulação e controle de peso), estimulação intelectual e socialização (Cordazzo \& Vieira, 2007; Melo \& Valle, 2005).

O convívio social é um ponto que merece destaque, pois está relacionado intimamente com a qualidade na variedade de estimulação. $\mathrm{Na}$ brincadeira é que o desenvolvimento tem a sua expressão máxima. Os jogos ou brincadeiras entre os pares possibilitam a criança vivenciar situações, realizar questionamentos e formular estratégias para superar determinado problema. É nesse momento que ela formula as relações de causa-efeito. Numa atmosfera com segurança emocional e ausência de perigo, a criança é quem vai encontrar soluções baseadas em seus erros e acertos, aprendendo, dessa maneira, as normas sociais vigentes no seu meio relacional. $\mathrm{O}$ aprendizado de viver socialmente e a forma de lidar com conflitos sociais também surgem durante a brincadeira (Cordazzo \& Vieira, 2007). A brincadeira, de certa forma, satisfaz as necessidades afetivas e permite lidar com maior habilidade sobre sentimentos de ansiedade e angústia. A família e o brincar desempenham o papel de potencializa- 
dores da aprendizagem e promovem a educação para os hábitos de vida diária (Cordazzo \& Vieira, 2007); além disso, servem de base para a assimilação da cultura e construção de valores sociais (Aranega et al., 2006).

A função do profissional da saúde é auxiliar a família a desempenhar seu papel, sem substituí-la, mas fornecendo o apoio necessário ao desenvolvimento de suas responsabilidades como tal através das intervenções. As intervenções devem ser realizadas em conjunto com a comunidade, estimulando sua mobilização e participação, com a finalidade de oferecer um serviço vinculado aos objetivos das Secretarias de Políticas de Saúde, assim se abalizam as limitações deste estudo que não teve a oportunidade de realizar intervenções com essas crianças estudadas. Sugere-se, para um próximo estudo, gerar orientações de como melhorar estes ambientes familiares para melhorar as oportunidades de desenvolvimento motor, bem como propor intervenções motoras para o mesmo fim.

\section{CONCLUSÕES}

Quanto maior o número de adultos na casa e o número de quartos, melhor o espaço físico. Quanto maior a escolaridade dos pais, melhor a oferta de brinquedos e materiais de motricidade fina. Quanto maior o número de crianças no domicílio melhor a variedade de estimulação. Quanto maior a renda familiar, maior é o espaço interior, espaço exterior, materiais de motricidade fina e de motricidade ampla. A criança que apresentou convívio social diário teve melhor variedade de estímulo. Embora as condições socioeconômicas adversas às comunidades estudadas, em geral, apresentaram um nível regular de oportunidades para o desenvolvimento motor das crianças, o convívio social obteve uma importante associação com a variedade de estimulação oferecida. Portanto, a criação de ambientes saudáveis, como praças, é de suma importância. A orientação dos pais e responsáveis pela criança a respeito das ativi- dades indicadas para determinada faixa etária também é muito importante, visto que eles podem ser multiplicadores desse pensamento.

\section{Agradecimentos:}

Nada declarado.

\section{Conflito de Interesses:}

Nada declarado.

\section{Financiamento:}

Nada declarado.

\section{REFERÊNCIAS}

Andrade, S. A., Santos, D. N., Bastos, A. C., Pedromônico, M.R.M., Almeida-Filho, N., \& Barreto, M.L. (2005). Ambiente familiar e desenvolvimento cognitivo infantil: Uma abordagem epidemiológica. Revista de Saúde Pública, 39(4), 606-611. doi: 10.1590/S003489102005000400014

Aranega, C. D. T., Nassim, C. P., \& Chiappetta, A. L. M. L. (2006). A importância do brincar na educação infantil. Revista CEFAC, 8(2), 141-146.

Brasil, Ministério da Saúde. (2000) Secretaria de Políticas de Saúde, Departamento de Atenção Básica. Programa Saúde da Família. Revista de Saúde Pública, 34(3), 316-319. doi: 10.1590/ S0034-89102000000300018

Bronfenbrenner, U. (2002). A ecologia do desenvolvimento humano: Experimentos naturais e planejados. Porto Alegre: Artes Médicas.

Cordazzo, S. T. D., \& Vieira, M. L. (2007). A brincadeira e suas implicações nos processos de aprendizagem e de desenvolvimento. Estudos e Pesquisas em Psicologia, 7(1), 92-104.

Gabbard, C., Caçola, P., \& Rodrigues, L. P. (2008). A new inventory for assessing Affordances in the Home Environment for Motor Development (AHEMD-SR). Early Childhood Education Journal, 36, 5-9. doi: 10.1007/S10643-008-0235-6

Gallahue, D. L., \& Ozmun, J. C. (2005) Compreendendo o desenvolvimento motor: Bebês, crianças, adolescentes e adultos. São Paulo: Phorte.

Maria-Mengel, M. R. S., \& Linhares, M. B. M. (2007). 
Risk factors for infant developmental problems. Revista Latino-Americana de Enfermagem, 15, 837-842.

Martins, M. F. D., Costa, J. S. D. da, Saforcada, E. T., \& Cunha, M. D. da C. (2004). Qualidade do ambiente e fatores associados: Um estudo em crianças de Pelotas, Rio Grande do Sul, Brasil. Caderno de Saúde Pública, 20(3), 710-718.

Melo, L. L., \& Valle, E. R. M. (2005). O brinquedo e o brincar no desenvolvimento infantil. Psicologia Argumento, 23(40), 43-48.

Monteiro, C. A., \& Freitas, I. C. M. (2000). Evolução de condicionantes socioeconômicas da saúde na infância na cidade de São Paulo (1984-1996). Revista de Saúde Pública, 34(6), 8-12.

Müller, A. B. (2008). Efeitos da intervenção motora em diferentes contextos no desenvolvimento da criança com atraso motor. Dissertação de Mestrado, Universidade Federal do Rio Grande do Sul, Porto Alegre, Rio Grande do Sul, Brasil.
Nicoletti, G., \& Manoel, E. J. (2007). Inventário de ações motoras de crianças no playground. Revista da Educação Física/UEM Maringá, 18(1), 17-26.

Papalia, D. E., \& Olds, S. W. (2000). Desenvolvimento humano. Porto Alegre: ARTMED.

Rodrigues, L., Saraiva, L., \& Gabbard, C. (2005). Development and construct validation of an inventory for assessing the home environment for motor development. Research Quarterly for Exercise and Sport, 76(2), 140-148.

Schobert, L. (2008). O desenvolvimento motor de bebês em creches: Um olhar sobre diferentes contextos. Dissertação de Mestrado, Universidade Federal do Rio grande do Sul, Porto Alegre, Rio Grande do Sul, Brasil.

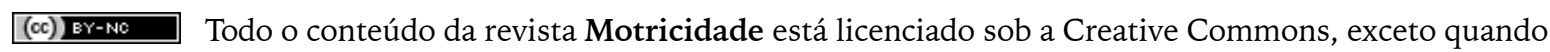
especificado em contrário e nos conteúdos retirados de outras fontes bibliográficas. 